\title{
Health Recommender System design in the context of CAREGIVERSPRO-MMD Project
}

\author{
Luis Oliva-Felipe \\ Universitat Politècnica de Catalunya - \\ BarcelonaTech \\ Barcelona, Catalunya, Spain \\ loliva@cs.upc.edu \\ Emma Wolverson \\ University of Hull \\ Hull, United Kingdom \\ e.wolverson@hull.ac.uk \\ Konstantinos Votis \\ Information Technologies Institute \\ Centre for Research and Technology \\ Hellas \\ Thessaloniki, Greece \\ kvotis@iti.gr
}

\author{
Cristian Barrué \\ Universitat Politècnica de Catalunya - \\ BarcelonaTech \\ Barcelona, Catalunya, Spain \\ cbarrue@cs.upc.edu \\ Marco Antomarini \\ COOSS Marche ONLUS \\ Ancona, Italy \\ m.antomarini@cooss.marche.it
}

\author{
Atia Cortés \\ Universitat Politècnica de Catalunya - \\ BarcelonaTech \\ Barcelona, Catalunya, Spain \\ acortes@cs.upc.edu \\ Isabelle Landrin \\ Centre Hospitalier Universitaire de \\ Rouen \\ Rouen, France \\ Isabelle.Landrin@chu-rouen.fr \\ Ulises Cortés \\ Universitat Politècnica de Catalunya - \\ BarcelonaTech \\ Barcelona, Catalunya, Spain \\ ia@cs.upc.edu
}

\begin{abstract}
CAREGIVERSPRO-MMD an EU H2020 funded project aims to build a digital platform focusing on people living with dementia and their caregivers, offering a selection of advanced, individually tailored services enabling them to live well in the community for as long as possible. This paper provides an outline of a health recommender system designed in the context of the project to provide tailored interventions to caregivers and people living with dementia.
\end{abstract}

\section{CCS CONCEPTS}

- Human-centered computing $\rightarrow$ Human computer interaction (HCI); Systems and tools for interaction design;

\section{KEYWORDS}

Recommender systems, Social Networks, Dementia, Caregiver

\section{ACM Reference Format:}

Luis Oliva-Felipe, Cristian Barrué, Atia Cortés, Emma Wolverson, Marco Antomarini, Isabelle Landrin, Konstantinos Votis, Ioannis Paliokas, and Ulises Cortés. 2018. Health Recommender System design in the context of CAREGIV MMD Project. In PETRA '18: The 11th PErvasive Technologies Related to Assistive Environments Conference, June 26-29, 2018, Corfu, Greece. ACM, New York, NY, USA, 8 pages. https://doi.org/10.1145/3197768.3201558

ACM acknowledges that this contribution was authored or co-authored by an employee, contractor or affiliate of a national government. As such, the Government retains a nonexclusive, royalty-free right to publish or reproduce this article, or to allow others to do so, for Government purposes only.

PETRA '18, June 26-29, 2018, Corfu, Greece

(C) 2018 Association for Computing Machinery.

ACM ISBN 978-1-4503-6390-7/18/06 ..\$15.00

https://doi.org/10.1145/3197768.3201558

\section{INTRODUCTION}

According to the World Health Organisation [31], 47M people around the world have some form of dementia, for which there is no effective intervention, to halt or reverse the progressive cognitive impairment. As Europe's population is ageing, long-term care for elderly citizens will become an increasing cost for society. To manage this transition, healthcare policies in the EU and individual Member States are heavily focused on extending the independent life of the elderly, with the dual aim of increasing their quality of life and reducing the costs of care.

Health delivery practices are shifting towards home care. The reasons are the better possibilities for managing chronic care, controlling health delivery costs, increasing quality of life and quality of health services and the distinct possibility of predicting and thus avoiding serious complications [17]. The concept of social networks using mobile devices to support healt communities has emerged as a new and viable reality in the field of IT for health and telemedicine under the funding of the EU programs. The so-called mHealth has the "potential to transform the face of health service delivery across the globe", and there is increasing media and consumer interest in RPiHealth, illustrated for example by a recent panel at the US Consumer Electronics Show on The Digital Health Revolution [19],[29], [30].

Ageing is one of the greatest social and economic challenges of the $21^{\text {st }}$ century for World societies. It will affect all EU countries and most policy areas. The Ageing of Europe is characterised by a decrease in fertility, a decrease in mortality rate, and a higher life expectancy among European populations, together with continued but decelerating inward net migration to the EU. In all Europe, life expectancy is increasing in an almost continuous and uniform trend at the rate of 2-3 months every year and is the main driver behind the population ageing. By 2025 more than $20 \%$ of Europeans 
will be 65 or over, with a particularly rapid increase in numbers of over-80s.

Due to an ageing population, the public provision of long-term care poses an increasing challenge to the sustainability of public finances in the EU. The ageing of the population is expected to put pressure on governments to provide long-term care services as old people often develop multi-morbidity conditions, which require long-term medical care and assistance with a number of daily tasks. Health trends among the elderly are mixed: severe disability is declining in some countries but increasing in others, while mild disability and chronic disease are generally increasing. This situation is comparable in many societies around the world.

CAREGIVERSPRO-MMD (C-MMD) is an EU funded project under the H2020 programme devoted to building a mHealth application that is specifically targeted to caregivers and people with cognitive impairment or mild to moderate dementia. The novelty of $C-M M D$ consists into integrating a broader diagnostic approach, incorporating the live-in family Caregiver-Person living with dementia dyad and considering this dyad as the unit of care.

CAREGIVERSPRO-MMD is focused on people living with Mild Cognitive Impairment or Mild to Moderate Dementia (PLWD from now on) and their caregivers. Mild Cognitive Impairment (MCI) is a condition that falls somewhere between normal age-related memory loss and Alzheimer's disease or a similar impairment. Not everyone with $M C I$ develops dementia. And like dementia, MCI is not an illness, but a cluster of symptoms that describe changes in how you think or process information. Memory problems are the most common indicators of $M C I$. A person with $M C I$ may also experience difficulties with judgement, orientation, thinking and language beyond what one might expect with normal ageing. For unknown reasons, $M C I$ appears to affect men more than women.

The project comprises three phases: first, to design and develop the first prototype of the mHealth application considering previous work on dementia and psychiatric co-morbidity symptoms, screening and intervention strategies to be implemented by the platform.

In the second phase, to conduct a user-centric analysis to redesign the existing prototype for PLWDs. The development was steered by PLWDs, caregivers and doctors, through user-centric design: feedback is being collected on each new version of the application until the design is adapted to the users' conditions.

In the third phase, the optimised application is being piloted with 600 dyads (PLWDs and their respective caregivers) and 600 controls. This will show the clinical and social benefits for PLWDs and caregivers, as well as financial benefits for the healthcare system.

$C-M M D$ is an intelligent support platform promoting Quality of Life (QoL), well-being and medication compliance for PLWD and Caregivers in the community at the point of care. It will be available on smartphones and tablet computer and web browsers. Its interface is being designed to suit users with low IT familiarity [20]. Additionally, $C-M M D$ will be compliant with internal security protocols and policies as well as industry regulatory policies, it is designed to only collect and process data concerning health for specific and legitimate purposes.

Some of the needs that PLWD and their informal caregivers currently perceive as insufficiently met by regular care and support services might be alleviated, or even be met with the help of the mutual assistance communities, using $C-M M D$. These needs can be summarized as $(i)$ the need for general and personalized information; (ii) the need for support with regard to symptoms of dementia; (iii) the need for social contact and company; and (iv) the need for health monitoring and perceived safety [14].

The therapeutic educational interventions is another $C-M M D$ 's important service. This service offers personalized educational contents to their PLWDs and caregivers through the social network feed tailored to their specific profile. The educational focus is twofold: first, it offers generic contents focused on dementia disease, dementia and psychiatric co-morbidity symptoms and available resources on the community and secondly, it proposes user-tailored content triggered on the basis of user's profile. In this paper, we will focus on this service provision.

\subsection{Plan of the work}

The plan for this paper is the following, in $§ 2$ we will explain the concept of intervention and the specific strategy selected in $C-M M D$. Section 3 provides background in recommender systems. In $\S 4$ we explain the recommender system approach developed in $C-M M D$. In $\S 5$ we will discuss our conclusions and comment the future work.

\section{PSYCHOSOCIAL INTERVENTIONS}

A health intervention is an act performed for, with or on behalf of a person or a population whose purpose is to assess, improve, maintain, promote or modify health, functioning or health conditions [22]. A broad range of providers can carry out interventions across the full scope of health and social systems including acute care, primary care, rehabilitation, and assistance with functioning, prevention and public health. Further definitions consider Educational Intervention as an intervention that aims to educate, inform and shape understanding of dementia and caregiving practice [9] and Psychosocial intervention as a broad term used to describe different ways to support people to overcome challenges and maintain good mental health $[7,8,10,23]$. In the literature, often the terms psychosocial and nonpharmacological are used synonymously to refer interventions. Psychosocial interventions are often administered by a trained person, e.g. a GP, a psychologist or psychotherapist, occupational therapists or nurses, and are usually person-centered. These sort of interventions are expected to help PLWD and often their caregivers as well, with [10]:

- coming to terms with a diagnosis of dementia

- maintaining social life and relationships after diagnosis

- reducing stress and improving mood, anxiety or depression

- reducing or adapting behaviour disorders

- improving cognitive functions, such as thinking and memory

- living independently

- maintaining and improving quality of life - maintaining health and happiness, and control over one's life

- supporting the partner and family

Historically, most psychosocial interventions have been administered in a face-to-face format between a provider (a trained person) and a care receiver (a PLWD, a Caregiver or both). More recently, these have also included the use of telephones or other digital devices, video conferences, self-guided books or Internet videos [21]. Some interventions combine one or more of these options. In the 
scope of C-MMD platform, the focus is on non-pharmacological educational interventions for PLWD and their caregivers that can be provided through digital means.

Tailored interventions, which are personalized to the individual and assessment based, take the concept one step further by adapting the intervention to specifically match the individual patient profile. This work describes the technical approach taken to implement the personalization of the contents presented to the $C-M M D$ platform users.

\section{$2.1 \quad C-M M D$ Interventions}

Interventions are well described in the literature but there is no widely accepted standard classification system that would allow for a more comprehensive understanding of the different types of treatments and care practices, the characteristics of people who benefit from them, or the kinds of problems each one has been shown to reduce or resolve [2]. However, many organisations provide their own proposals under different criteria. The NICE-SCIE, for instance, categorizes interventions according to their use: cognitive symptoms, non-cognitive symptoms and behaviour, comorbid emotional conditions and caregiver support. On the other hand, other classifications can also identify interventions for the problem they target (e.g. sleep problems). In the $C-M M D$ ecosystem two core groups of stakeholders can be found, divided their knowledge background: (i) Health and Social Professionals (HSP) and (ii) Caregivers/PLWD. The importance of developing and using language and terminology that is understandable and meaningful to these stakeholder groups in all efforts to expand the availability and use of non-pharmacological treatments and care practices must be emphasized. The $C-M M D$ platform must allow the HSP to classify correctly the interventions and contents they create for the PLWD and Caregivers while these must be able to identify their problems and needs in the categorisation. The $C-M M D$ Consortium has explored several intervention taxonomies from trusted sources like:

- Interdem, a pan-European network of researchers collaborating in research on and dissemination of Early, Timely and Quality Psychosocial Interventions in Dementia [18]

- The National Institute for Health and Clinical Excellence Social Care Institute for Excellence Guideline on Supporting People With Dementia and Their Carers in Health and Social Care [9]

- The British Psychology Society Guide to Psychosocial Interventions in Early Stages of Dementia [10]

- Dementia Carer site developed with and by family members who look after someone who has dementia [28]

The $C-M M D$ Consortium has identified the domains of the Dementia Carer proposal as the more significant, as they reflect the most the point of view of dyad daily life but it must be noted that there is a consistent parallelism in the different proposals. Based on literature survey, the $C-M M D$ Consortium depicted a list of 26 interventions categorized by intervention area from which 12 were selected (see Table 1) to be as candidates to be integrated into the $C$ $M M D$ platform upon the feasibility to be technically implemented.

\subsection{Intervention Provision}

According to NICE-SCIE, treatment and care should take into account patients' needs and preferences (or healthcare goals). People with dementia should have the opportunity to make informed decisions about their care and treatment, in partnership with their healthcare professionals. One of the main characteristics of the $C-M M D$ platform is the possibility to empower both the PLWD and the Caregivers improving the abilities for the self-management of their health and taking into account their attitudes and inclination. For such reason, the strategy of intervention provision is also established according to the user's preferences. One of the $C-M M D$ primary services is to provide user-tailored interventions, ranked according to users' needs and preferences and screening results. In order to provide tailored interventions, it has to be taken into account these two important dimensions: user preferences and the users' clinical/social profile. Person-defined healthcare goals and preferences align primary and speciality care focusing on what matters most to the people. PLWDs and Caregivers should be far more activated and engaged in their care when it focuses on achieving what matters most to them.

The main goal of the content recommendation system will be to combine the user preferences (based on their interests), the healthcare professional recommendations (based on their professional criteria) and the Caregivers' opinion (considering their care experience):

- A PLWD can select its topic preferences when registering

- A Caregiver can define its topic preferences when registering and also suggest topics for its cared one considering his/her specific needs

- A healthcare provider (medical/social) can define topic goals for his managed PLWD/Caregiver considering his/her specific needs

- Other healthcare metadata related to the user profile is used to recommend interventions

All this preference selection is dynamic and can be modified in the system at any time, allowing a flexible personalization.

\section{RECOMMENDER SYSTEMS BACKGROUND}

$C-M M D$ platform will take advantage of recommender system technologies in order to provide PLWD and Caregivers with a personalized list of psychosocial interventions. This section provides an overview of the related work and methods that have been investigated to develop the Health Recommender System for the $C-M M D$ project.

In the mid-nineties of last century, Recommender Systems emerged as an independent research field of Information Retrieval and Artificial Intelligence to address the information overload problem by using a specific type of information filtering techniques that attempts to recommend information items (e.g., movies, TV, videos on demand, music, books, news, images, Web pages, research papers) that are likely to be of interest to the user. In public health systems, not only there is an overload problem but also information comes from different sources and formats. Personal health record systems are meant to centralise and standardise an individual's health data and enhance the data sharing among authorised professionals or entities. Therefore, recent trends in research have focused 
Table 1: C-MMD Intervention Types

\begin{tabular}{|c|c|}
\hline Domain & Intervention \\
\hline \multirow{3}{*}{ Information and adjustment to diagnosis } & Dementia Advisors \\
\hline & Post Diagnostic Groups \\
\hline & Signposting \\
\hline \multirow{3}{*}{ Stress, anxiety or depression management } & Peer Support Groups \\
\hline & Stress/Anxiety management \\
\hline & Reminiscence \\
\hline \multirow{2}{*}{ Improving and maintaining cognitive functioning } & Assistive Technology: advice and support \\
\hline & Cognitive Training \\
\hline \multirow{4}{*}{ Health and quality of life } & Physical Exercise therapy \\
\hline & Home modification \\
\hline & Fall prevention \\
\hline & Music Therapy \\
\hline
\end{tabular}

on health information retrieving and different approaches to Health Recommender Systems (HRS) can be found in the literature.

An HRS is a specialisation of a recommender system, where a recommendable item of interest is "a piece of non-confidential, scientifically proven or at least generally accepted medical information, which in itself is not linked to an individual's medical history" [32]. Possible examples of a recommended item are food/nutrition information, a physical activity, a diagnosis, a therapy or a medication [5]. Authors in [32] have identified HRS designed for two target publics and purposes :

- diagnostic or educational tool to assist physicians in the decision-making process when treating a patient

- personal health advising tool for users, especially focused on healthy behavioural change, engaging users into physical activities or nutrition based recommender systems

The final objective is to empower patients by educating them about their health and offering means to self-diagnosis, but also to provide clinicians with more accurate information about his/her patients. Finally, health care providers or insurances would benefit from this efficient, tailored and cost-saving services [27]. One of the main challenges for HRS, in comparison to the ones faced by traditional recommender systems, is the implication of ethical issues when collecting or processing personal data and the health information delivered to the end-user. People might present different, multiple conditions, and each of them will have different needs and interests. HRS ought to be able to determine these health conditions and which is the meaningful data from the patients' health record, in order to provide tailored, context-related, high quality and trusted recommendations.

Nowadays, HRS are becoming popular due to the increasing demand of nutrition-based and healthier lifestyle behavioural recommenders. The Intrapersonal Retrospective Recommendation (IRR, [16]) is a lifestyle change recommender that only uses personal history and the goal of achievement. This approach might suffer from the new user problem since recommendations are based on the retrospective history and behavioural patterns of an individual. MyBehaviour [24] is a mobile application which tracks a user's physical activity and diet routines and provides automatic health feedback.
Educational HRS is the other main field of application. An example of it is HealthRecSys [26], a semantic-based recommender which generates Medline Plus links extracted from metadata of selected Youtube videos. Authors remark that the quality of recommendations is affected by the semantic-gap between the layperson language and the health professional.

In [11], authors propose a recommender system that combines both approaches to develop a smartphone-based personalized recommender system for people with depression. The system will both propose activities to relieve negative emotions, but also offers expert knowledge on how to be aware of such negative emotions and to help users learn how to contain and change them.

In general, HRS are still in an immature phase although it is an emerging trend in literature. In [5], authors proposed a framework for HRS where all the components needed for creating a successful and useful recommender system are described: (i) domain definition, including items, context, stakeholders, end-users or data availability, (ii) HRS evaluation, particularly user acceptance and satisfaction, trust and privacy, and communication quality, (iii) behavioural evaluations, or how effective was the HRS, (iv) health impact, which assesses how behavioural changes lead to changes in health and $(v)$ ethical considerations of the HRS.

\subsection{Recommendation strategies}

In the literature [25], recommendation strategies are classified either on the basis of their knowledge source or of the algorithmic technique employed. When classified on the basis of the knowledge source, the following two main strategies can be identified: contentbased filtering (CB), which expresses user interests as keywordbased user profiles and consists of recommending items matching user preferences and item features; and collaborative filtering $(\mathrm{CF})$, which expresses user preferences as item ratings and whose recommendations are based on matching users or items with similar rating behaviour. Based on the algorithmic technique, two main approaches can be identified as well: heuristic-based (also called memory-based), which employs some heuristic formula, such as vector-based similarity and correlation measures, to calculate the recommendation; and model-based, which generates the recommendation using a model learnt by applying some model-building 
technique over the user-item rating matrix. Typically, heuristicbased systems adapt better to changes in user's preferences but scale worse than model-based ones. Heuristic-based CB [1] is inspired by Information Retrieval methods and calculates the user-item matching based on vector measures such as the cosine similarity. Model-based CB [3] calculates the matching based on a model of user's tastes built by applying Machine Learning techniques, such as naïve Bayesian networks, to text categorization. Heuristic-based $\mathrm{CF}$ employs heuristic techniques such as rating correlation analysis and is also known as the neighbourhood-based approach (based on the k-nearest-neighbour algorithm). Depending on whether a subset of users or items is chosen to compute recommendations, two classes of heuristic-based CF can be identified: user-based [12], when the algorithm focuses on finding users similar to the active one for recommending; and item-based [15], when the algorithm focuses on finding items similar to those the active user likes. Modelbased $\mathrm{CF}$ uses the ratings to learn a compact predictive model to represent user-item interactions based on latent factors [13]. In general, recommender systems have a significant dependency on data. It is possible to distinguish the following problems:

(1) Cold-start problem: To provide good recommendations it is necessary to have enough feedback from users (e.g., ratings of previously delivered interventions) to learn which items fit their needs. For this reason, new users represent a blank slate and the system does not know how to provide personalised results since there are no elements of the decision to do filtering. This situation is also known as the cold start problem and it also affects items. It can be usually found when using collaborative filtering. In this situation, a new item does not have any rating.

(2) Sparsity: Usually, the set of items grows larger than the set of users, which implies that as the system gets older, the average number of ratings by item becomes lower, since users tend to rate a limited number of items. The effect of this situation is that users become less similar since there are less common ratings between them.

(3) Generalization: The generalization problem arises when the user model is too generic, and it does not allow to make personalized recommendations but just in a generic way, thus little differences can be found from what is recommended between different users

(4) Overspecialization: Opposed to generalization, this issue occurs when the recommender system collects a significant amount of feedback too focused on a subset of items. In that case, the system may just recommend those items that are high likely to be already known by the user and ignoring the rest.

(5) Insensitiveness to preference changes: If the user model is not learnt periodically, it can turn out-dated and do not offer recommendations aligned with current user's preferences.

In addition to this, it is possible to find Demographic-based approaches (DF), which build stereotypes according to users' aggregated personal details, thus generating user models from similar users' groups (with regards demographic criteria such as age or gender).
Table 2: Trade-offs in recommendation strategies

\begin{tabular}{llll}
\hline Limitations of Recommendation Strategies & CB & CF & DF \\
\hline New user cold-start problem & X & X & \\
New item cold-start problem & & X & X \\
Sparsity problem & & X & X \\
Generalization problem & X & X \\
Overspecialization problem & X & & \\
Insensitive to preference changes & & X & X \\
\hline
\end{tabular}

Each algorithmic approach has proved to obtain better results to solve some, but not all, of them (see Table 2). In isolation, CF has proven to be the most accurate approach in most domains. However, these methods suffer from well-known limitations that considerably affect their performance. The most common solution to overcome these limitations in real applications consists of applying a hybrid strategy combining $\mathrm{CB}$ and $\mathrm{CF}$ methods. Different hybridization strategies have been used in the literature, such as: the weighted strategy, in which the score of different recommendation components are combined numerically; the feature augmentation strategy, in which the recommendation technique is used to compute a feature or set of features, which is then part of the input to another technique; and the cascade strategy, in which recommendations made by one technique are refined by another technique. For instance, using DF approach to deal with cold-start problems of new users, where nothing is known about the user and, at least, some approximation can be performed to offer a user model more useful than just randomly recommending. Afterwards, with more knowledge about users, a more refined user model can be learnt.

\section{C-MMD RECOMMENDER COMPONENT}

This section presents an overview of the undertaken solution approach to design the Health Recommender System. This system provides two different services: a ranked list of interventions which is tailored to user's preferences and requirements and a list of potential acquaintances according to their similarities to the given user. Although both functionalities provided by the system share similar components and interfaces towards data sources, their objectives and the nature of the items that each one handles, differs. For this reason, each part has been designed differently: their training and recommendation processes, although basically similar, have some slight differences. It is worth noting that the former has already been implemented while the latter has not been yet deployed at the moment of writing this paper. Nevertheless, we will mention this second recommender along the text as it has affected the overall design of the system.

\section{Algorithmic approach}

To develop the $C$-MMD HRS we adopted a hybrid filtering approach. It is well known that hybrid systems provide better results [4], consequently, that is the chosen approach. Specifically, our approach consists of a content-based algorithm and a rule-based filtering which uses a taxonomical classification of interventions used by health professionals that create those interventions. In this way, we aim to provide medical criteria for the process of selecting what 
interventions should be returned to the user. Ideally, there is a large number of possible interventions, as many as the different kinds of subjects, areas of interest and so that a user can indicate in its profile. It is also necessary to note that given the nature of the users, it may be possible that not too much feedback can be collected in terms of ratings. This implies that any feedback retrieved from users may be too sparse, which makes difficult to find similar users to proceed with a collaborative-filtering approach. These reasons strengthen our approach of applying a content-based algorithm to predict user's satisfaction along the use of a rule-based approach which takes into consideration the topics of interest chosen by the PLWD, caregiver and doctors/social workers. By means of extracting features that define an intervention, it is possible to learn the preference towards each feature from user's feedback given to previous interventions. For instance, a user may provide positive feedback towards interventions that are mainly videos, explaining tips on a specific topic of interest, and negative feedback to interventions that are long texts. Thus, a content-based algorithm can predict better score to interventions that are delivered in video format. Besides this, the recommender system also computes a list of similar users for a given user. This list is understood as potential acquaintances or friends for that user, which are relevant because of the shared interests they have. Feedback is collected through the $C-M M D$ platform and its graphical interface. We can distinguish two different kinds of feedback:

(1) Explicit: Feedback that the user has explicitly provided, through the $C-M M D$ graphical interface, and includes User's preferences and personal details. It also includes explicit ratings given to interventions. Explicit feedback can become inaccurate or out-dated since it depends on the user to actively fill in all information as well as update it. This is especially true for PLWD users; thus, we also include the preferences suggested by caregivers and doctors/social workers with regards to their related PLWD

(2) Implicit: Feedback that user has not actively provided, but it has been obtained from the actions $\mathrm{s} / \mathrm{he}$ has carried out and it is captured automatically by the platform (e.g., interventions viewed or shared)

Our initial design is using both kinds of feedback. Specifically, it is using implicit feedback in terms of interventions that have been effectively consumed by the user. This feedback is obtained through the feedback interface. Our system is also using explicit feedback in terms of likes/dislikes that a user may have provided towards interventions. It was also considered to ask users to rate using a 1-5 Likert scale but it was discarded since using this more fine-grained rating could difficult user experience given the nature of their illnesses.

\section{Implementation details}

The HRS system is composed of the following components (see figure 1):

(1) The HRS manager, who takes care of orchestrating the learning process, combining the results of the predictions made by both recommender systems, apply hard constraints as pre or post filtering rules
(2) The recommender systems, that is, the approaches were taken to learn user models and predict the ratings on nonconsumed interventions as well as similar users

(3) The adaptors, meant to decouple the internal data models of the recommender from the data sources models provided by other components in the CMMD platform, thus containing potential problems from changes in those data model schemas

(4) An endpoint to provide the recommendations

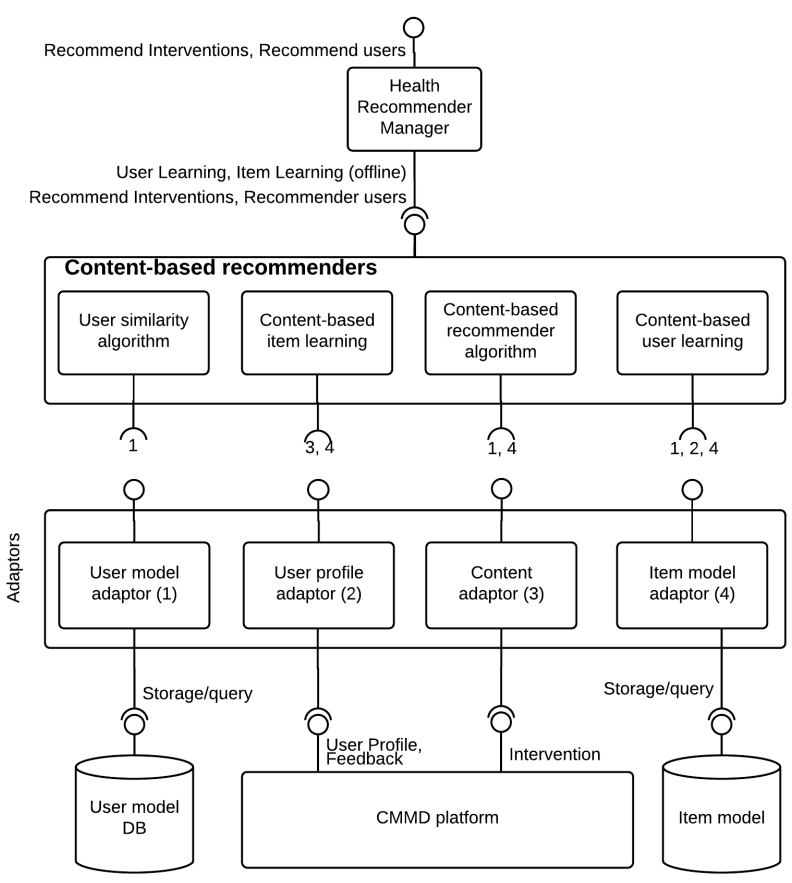

Figure 1: HRS architecture overview. Each element in the content-based recommenders box can consume from different adaptors.

The previous components work offline; the user models require being updated and re-learnt from time to time. The update frequency is currently set in a daily basis, but it may be changed depending on the frequency that users rate the items (interventions) or change their preferences. The system computes predictions and stores them to be accessed later on through an API that publishes them for each user. This HRS system has been implemented in Java and Clojure. The former takes care of the engine internals while the latter wraps the engine to take care of the public interface and data preparation. We have used two different languages so we can use Java to get the advantage of existing libraries (e.g., Apache Mahout) and Clojure. Using Java also facilitates reusing the code more easily in different scenarios and deployments as well as maintaining it in the future. Clojure smoothly interoperates with Java (both are executed in the JVM) and facilitates the task of providing a selfdocumented RESTful API as well as data cleansing and preparation for the learning process performed by the Java code. 
As mentioned before, the HRS implements a content-based approach which has been refined with pre-filtering and post-treatment processes. The former removes those interventions that are irrelevant to the user according to the interests declared by the user, his/her caregiver and doctor and his/her profile (e.g. language, comorbidities, location). Afterwards, the system predicts the estimated interest of the user towards the potential intervention candidates according to user's personal data (e.g., user's details), user-generated content (i.e., ratings, likes, shares of content that was recommended). The last step post-processes this list to rank them according to user's interests and user's caregiver and doctor indications.

In this way, the user is receiving a list of interventions which are grouped according to not only on user's interests but also on what his/her doctor and caregiver consider more useful or suitable and, within each group, ranked to the estimated preference of the user towards those interventions.

The learning process is performed daily and the result is stored for consumption by the $C-M M D$ platform which schedules the appearance of recommended interventions in user's news feed throughout the day, thus constantly providing interesting interventions to keep user's attention.

\section{CONCLUSIONS}

In this paper, we have outlined the design of an HRS for the $C$ $M M D$ platform that is already being piloted by 600 dyads in four countries. The data collected the next months will allow us to fine-tune the recommending process and to provide results on the content distribution and consumption by the different stakeholders.

Elderly people with cognitive impairment feel there is a great need for more public awareness of the disease and more support for caregivers. While treatments to reverse or halt disease progression are not available for most of the dementias, and for the foreseeable future, treatment and medication of dementia will remain centred entirely on disease management. Slowing down the rate of cognitive decline and improving early diagnosis is essential in this as it gives PLWDs the best chance to maintain their cognitive ability. It also allows caregivers and PLWDs to plan for the future. $C-M M D$ supports PLWDs and Caregivers, providing relevant interventions for them at the point of care in the community, empowering them by building up patients' capacity for a better self-management of the disease and to become active partners in their own care, and to contribute to a wider perspective in the health care system.

\subsection{Future Work}

Next development steps in the recommender component are headed towards the implementation of a second recommender functionality to provide a list of potentially interesting acquaintances for a given user. This will support the social network that Caregivers platform is aimed to build. This list will be based on users' similarities based on interests and interactions with previously recommended interventions and also based on users' profiles (e.g., screening results, comorbidities, language).

\section{ACKNOWLEDGMENTS}

The work presented in this paper has been performed under the European Project CAREGIVERSPRO-MMD[6], which has received funding from the European Union Horizon 2020 research and innovation programme under grant agreement No 690211.

Prof. Ulises Cortés is a member of Sistema Nacional de Investigadores (SNI-III), México.

\section{REFERENCES}

[1] Jae-wook Ahn, Peter Brusilovsky, Jonathan Grady, Daqing He, and Sue Yeon Syn. 2007. Open user profiles for adaptive news systems: help or harm?. In Proceedings of the 16th international conference on World Wide Web. ACM, 11-20.

[2] Administration on Aging" "Alliance for Aging Research. "2012". "Translating Innovation to Impact: Evidence-based interventions to support people with Alzheimer's disease and their caregivers at home and in the community". "MetLife Foundation".

[3] Daniel Billsus and Michael J Pazzani. 1999. A hybrid user model for news story classification. In UM99 User Modeling. Springer, 99-108.

[4] Robin Burke. 2007. Hybrid web recommender systems. In The adaptive web. Springer, 377-408.

[5] André Calero Valdez, Martina Ziefle, Katrien Verbert, Alexander Felfernig, and Andreas Holzinger. 2016. Recommender Systems for Health Informatics: State-ofthe-Art and Future Perspectives. Springer International Publishing, Cham, 391-414. https://doi.org/10.1007/978-3-319-50478-0_20

[6] CAREGIVERSPRO-MMD Consortium. 2018. CAREGIVERSPRO-MMD. (2018). Retrieved March 2, 2018 from https://caregiversprommd-project.eu

[7] Grupo de trabajo de la Guía de Práctica Clínica sobre la atención integral a las personas con enfermedad de Alzheimer y otras demencias. 2010. Guía de Práctica Clínica sobre la atención integral a las personas con enfermedad de Alzheimer y otras demencias. Agencia d'Informació, Avaluació i Qualitat en Salut de Catalunya;.

[8] Karine Petitprez et al. 2011. Maladie d'Alzheimer et maladies apparentées : diagnostic et prise en charge. Recommendation de bonne pratique. Haute Autorié de Santé.

[9] National Institute for Health and Clinical Excellence Social Care Institute for Excellence. 2007. A NICE-SCIE Guideline on supporting people with dementia and their carers in health and social care. The British Psychological Society and Gaskell.

[10] Reinhard Guss and colleagues. 2014. A Guide to Psychosocial Interventions in Early Stages of Dementia,. The British Psychological Society.

[11] Galen Chin-Lun Hung, Pei-Ching Yang, Chen-Yi Wang, and Jung-Hsien Chiang. 2015. A Smartphone-Based Personalized Activity Recommender System for Patients with Depression. In Proceedings of the 5th EAI International Conference on Wireless Mobile Communication and Healthcare (MOBIHEALTH'15). ICST (Institute for Computer Sciences, Social-Informatics and Telecommunications Engineering), ICST, Brussels, Belgium, Belgium, 253-257. https: //doi.org/10.4108/eai.14-10-2015.2261655

[12] Joseph A Konstan, Bradley N Miller, David Maltz, Jonathan L Herlocker, Lee R Gordon, and John Riedl. 1997. GroupLens: applying collaborative filtering to Usenet news. Commun. ACM 40, 3 (1997), 77-87.

[13] Yehuda Koren and Robert Bell. 2011. Advances in collaborative filtering. In Recommender systems handbook. Springer, 77-118.

[14] Steve Lauriks, Annika Reinersmann, Henriëtte Geralde Van der Roest, FJM Meiland, Richard J Davies, Ferial Moelaert, Maurice D Mulvenna, Chris D Nugent, and Rose-Marie Dröes. 2007. Review of ICT-based services for identified unmet needs in people with dementia. Ageing Research Reviews 6, 3 (2007), 223-246.

[15] Greg Linden, Brent Smith, and Jeremy York. 2003. Amazon. com recommendations: Item-to-item collaborative filtering. IEEE Internet computing 7, 1 (2003), 76-80.

[16] Bernd Ludwig, Francesco Ricci, Zerrin Yumak, Nava Tintarev, Rong Hu, Pearl $\mathrm{Pu}$, Efthimios Bothos, Dimitris Apostolou, Gregoris Mentzas, Robert G. Farrell, Catalina Danis, Sreeram Ramakrishnan, Wendy A. Kellogg, Ugan Yasavur, Reza Amini, and Christine L. Lisetti. 2012. Intrapersonal Retrospective Recommendation: Lifestyle Change Recommendations Using Stable Patterns of Personal Behavior.

[17] Nicos Maglaveras, Vasilis Koutkias, Ioanna Chouvarda, Dimitrios G. Goulis, A. Avramides, D. Adamidis, George Louridas, and EA. Balas. 2002. Home care delivery through the mobile telecommunications platform: the Citizen Health System (CHS) perspective. Int 7 Med Inform. 68, 1-3 (2002), 99-111.

[18] Esme Moniz-Cook, Myrra Vernooij-Dassen, Bob Woods, Martin Orrell, and Interdem Network. 2011. Psychosocial interventions in dementia care research: The INTERDEM manifesto. Aging \& Mental Health 15, 3 (2011), 283-290. https://doi.org/10.1080/13607863.2010.543665 arXiv:https://doi.org/10.1080/13607863.2010.543665 PMID: 21491215.

[19] Salla Muuraiskangas, Marja Harjumaa, Kirsikka Kaipainen, and Miika Ermes. 2016. Process and Effects Evaluation of a Digital Mental Health Intervention targeted at Improving Occupational Well-being: Lessons From an Intervention Study With Failed adoption. FMIR Ment Health 3, 2 (2016). https://doi.org/doi: 10.2196/mental.4465 
[20] OECD. [n. d.]. Skills Outlook 2013: First Results from the Survey of Adult Skills ([n. d.]).

[21] Institute of Medicine. 2015. Psychosocial Interventions for Mental and Substance Use Disorders: A Framework for Establishing Evidence-Based Standards. The National Academies Press, Washington, DC. https://doi.org/10.17226/19013

[22] World Health Organisation. 2016. International Classification of Health Interventions. (2016). Retrieved March 2, 2018 from http://www.who.int/classifications/ ichi/en/

[23] Ronald C. Petersen, J.C. Stevens, Mary Ganguli, Eric G. Tangalos, J.L. Cummings, and Steven T. DeKosky. 2001. Practice parameter: Early detection of dementia: Mild cognitive impairment (an evidence-based review) Neurology 56, 9 (2001), 1133-1142. https://doi.org/10.1212/WNL.56.9.1133 arXiv:http://n.neurology.org/content/56/9/1133.full.pdf

[24] Mashfiqui Rabbi, Min Hane Aung, Mi Zhang, and Tanzeem Choudhury. 2015 MyBehavior: automatic personalized health feedback from user behaviors and preferences using smartphones. In UbiComp.

[25] Francesco Ricci, Lior Rokach, and Bracha Shapira. 2011. Introduction to recommender systems handbook. In Recommender systems handbook. Springer, $1-35$.

[26] Carlos Luis Sanchez Bocanegra, Jose Luis Sevillano Ramos, Carlos Rizo, Anton Civit, and Luis Fernandez-Luque. 2017. HealthRecSys: A semantic contentbased recommender system to complement health videos. BMC Medical Informatics and Decision Making 17, 1 (15 May 2017), 63. https://doi.org/10.1186/ s12911-017-0431-7

[27] Hanna Schäfer, Santiago Hors-Fraile, Raghav Pavan Karumur, André Calero Valdez, Alan Said, Helma Torkamaan, Tom Ulmer, and Christoph Trattner 2017. Towards Health (Aware) Recommender Systems. In Proceedings of the 2017 International Conference on Digital Health (DH '17). ACM, New York, NY, USA, 157-161. https://doi.org/10.1145/3079452.3079499

[28] Dementia Carer Team. 2018. Dementia Carer. (2018). Retrieved March 1, 2018 from http://www.dementiacarer.net/

[29] The PLOS Medicine Editors. 2013. A Reality Checkpoint for Mobile Health Three Challenges to Overcome. PLoS Medicine 10, 2:e1001395 (2013). https: //doi.org/doi:10.1371/journal.pmed.1001395

[30] WHO. 2013. Call for innovative health technologies. (2013). Retrieved March 1, 2018 from http://www.who.int/ehealth/en/

[31] WHO. 2016. Dementia Fact sheet. (2016). Retrieved March 1, 2018 from http://www.who.int/mediacentre/factsheets/fs362/en/

[32] Martin Wiesner and Daniel Pfeifer. 2014. Health Recommender Systems: Concepts, Requirements, Technical Basics and Challenges. International fournal of Environmental Research and Public Health 11, 3 (2014), 2580-2607. https: //doi.org/10.3390/ijerph110302580 